\title{
Durchbruch
}

\section{für die Sozialwirtschaft}

\author{
Eberhard Eichenhofer
}

Der Ausschuss für Beschäftigung und soziale Angelegenheiten des Europäischen Parlaments hat Anfang des Jahres einen "Bericht über die Sozialwirtschaft « verabschiedet. Das Dokument ist für die Sozialwirtschaft von weitrechender Bedeutung. In ihm findet die Sozialwirtschaft als eine innovative, dem Gemeinwohl verpflichtete Institution eine umfassende Anerkennung. Der Bericht öffnet vielfältige Perspektiven für die Fortentwicklung des europäischen Rechts.

Die Berichterstattung des Europäischen Parlaments versteht sich vor dem Hintergrund der in Art. 16 EGVertrag (EGV) und Art. 36 EU-Grundrechte-Charta geltenden Regeln. In Art. 16 EGV anerkennt die Europäische Gemeinschaft (EG) die Dienste von allgemeinem Interesse (Universaldienste) und bekräftigt deren Ausnahme von einzelnen Bestimmungen des EG-Wirtschaftsrechts (Art. 73, 86 f. EGV). Ferner wird betont, dass Universaldienste die Werte der EG verwirklichen und den wirtschaftlichen und sozialen Zusammenhalt vertiefen. Schließlich begründet Art. 16 EGV für die EG wie die Mitgliedstaaten die Pflicht, dass beide »Grundsätze und Bedingungen für das Funktionieren der Dienste so gestalten, dass die Dienste ihren Aufgaben nachkommen können«. Nach Art. 36 der derzeit noch unverbindlichen - EUGrundrechte-Charta steht jedem Menschen ein Zugang zu den Universaldiensten im Rahmen der in den einzelnen Mitgliedstaaten vorherrschenden Gepflogenheiten zu; auch diese Bestimmung unterstreicht die Bedeutung der Universaldienste zur Vertiefung des wirtschaftlichen und sozialen Zusammenhalts.
Der Vertrag von Lissabon bekräftigt diese Grundsätze und macht die Gewährleistung des Zugangs zu Universaldiensten verpflichtend und verbindlich. Außerdem werden Rat und Europäisches Parlament dazu verpflichtet - unbeschadet der Rechte der Mitgliedstaaten hinsichtlich der Errichtung und Finanzierung der Einrichtungen der Universaldienste - die »Grundsätze und Bedingungen ihres Wirkens« durch Verordnung festzulegen (Art. 14 AEUV). Die Ausgestaltung der Sozialwirtschaft in den Grundzügen ihrer Betätigung wird daher im künftigen Primärrecht eine anerkannte Materie europarechtlicher Gesetzgebung werden. Im Hinblick darauf enthält der Bericht durchaus Eckpunkte und Rahmenbedingungen für eine mögliche künftige europaweite Normierung der Sozialwirtschaft. Vor diesem Hintergrund kommt dem Bericht eine beträchtliche rechts- und sozialpolitische Bedeutung zu.

\section{Kernaussagen des Berichts}

Das Europäische Parlament stützt seine Initiative auf die der EG zukommenden sozial- und beschäftigungspolitischen Befugnisse sowie deren Recht zur Normsetzung auf dem Gebiete des grenzüberschreitenden Gesellschaftsrechts (Art. 3, 48, 125-130, $136 \mathrm{EGV})$. Es verweist ferner auf zahlreiche, im Einzelnen aufgeführte und in den vergangenen Jahrzehnten ergriffene Initiativen zur Förderung und Ausgestaltung der Sozialwirtschaft auf EG-Ebene.

Das Dokument hebt sodann die zentrale Rolle hervor, welche der Sozialwirtschaft im Rahmen des Europäischen Sozialmodells zukommt

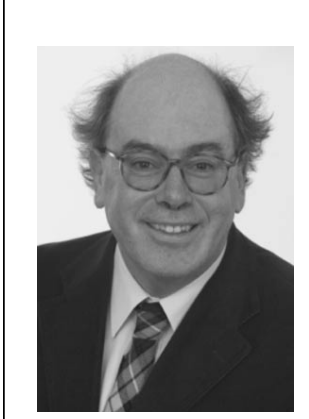

Prof. Dr. Dr. h.c. Eberhard Eichenhofer hat Rechts- und Politikwissenschaft an den Universitäten Tübingen und Saarbrücken studiert. Bevor er 1997 den Lehrstuhl für Sozialrecht und Bürgerliches Recht an der Friedrich-Schiller-Universität Jena übernahm, lehrte er an der Universität Osnabrück. Der vorliegende Beitrag basiert auf seiner Expertise im Auftrag des Brüsseler Kreises zum Bericht des Europäischen Parlaments verfasst. Der Brüsseler Kreis ist ein Zusammenschluss großer evangelischer und katholischer Sozialunternehmen in Deutschland. Mit rund 40.000 Mitarbeitenden und mehr als 35.000 stationären, teilstationären und ambulanten Angeboten erreicht der Brüsseler Kreis jährlich rund 100.000 Klienten (weitere Informationen: http://www.bruesseler-kreis.de).

E-Mailee@recht.uni-jena.de

und zufällt: ihr innovatives Potenzial, ihr soziales Paradigma sowie ihr Wachstums-, Beschäftigungs- und Bildungspotential, die Vielfalt ihrer Organisationsformen, ihrer Ausrichtung auf das Ziel der sozialen Eingliederung, Gleichstellung der Geschlechter, Schutz der älteren, behinderten und älteren Menschen, ihre hohe wirtschaftliche und Be- 
schäftigungsfunktion, ihre Ausrichtung auf kleine, mittlere Unternehmen, ihre eigenen Organisationsformen sowie ihre Verpflichtung gegenüber dem Allgemeinwohl. Aus diesem allem folgt der hohe Wert der Sozialwirtschaft für die Wirtschaftsordnung der EG. In ihrer Verknüpfung von Wirtschaftlichkeit und Gemeinwohlbindung wird der Sozialwirtschaft bescheinigt, qualitativ gute Arbeitsplätze zu schaffen, den sozialen, wirtschaftlichen und regionalen Zusammenhalt zu vertiefen, soziales Kapital zu bilden, »eine Art von Wirtschaft mit demokratischen Werten« zu fördern, weil sie auf den umfassend zu fördern. Dafür sei insbesondere ein erleichterter Zugang zu Krediten und Steuervergünstigungen notwendig.

Um einen sicheren Rechtsrahmen zu schaffen, müsste die Anerkennung der sozialwirtschaftlichen Unternehmungen in allen mitgliedstaatlichen Rechtsformen gewährleistet werden. Entsprechende Bestrebungen der Kommission zur Schaffung einer europäischen Gesellschaft auf Gegenseitigkeit und eines europäischen Vereins sind zum Bedauern des Europäischen Parlaments nicht weiter verfolgt worden. Initiativen zur

\section{»Das Europäische Parlament sieht in der Sozialwirtschaft eine Wirtschaft mit demokratischen Werten. «}

\begin{abstract}
Menschen und die nachhaltige Entwicklung der Gesellschaft ausgerichtet ist. Die Sozialwirtschaft stärkt auch die Erwerbswirtschaft und zwar sowohl durch ihre Leistungen, als auch symbolisch.
\end{abstract}

Das Europäische Parlament gibt sodann seiner Überzeugung Ausdruck, dass die europäische Politik ihren Beitrag zur vollen Entfaltung des gesamten Potentials der Sozialwirtschaft unter Wahrung der Vielfalt der Organisationsformen in den Mitgliedstaaten zu leisten habe. Dies verlange nach einem eigenen und sicheren Rechtsrahmen, in dem die Sozialwirtschaft von einzelnen Wettbewerbsvorschriften auszunehmen sei. Es wird ferner darauf hingewiesen, dass sozialwirtschaftliche Unternehmungen weit weniger als erwerbswirtschaftliche Unternehmen von den unvorhersehbaren Entwicklungen der Kapitalmärkte abhängen.

Unter Berufung auf die Anerkennung der Vielfalt der Gesellschaftsformen im EG-Vertrag fordert das Europäische Parlament die Kommission auf, die Sozialwirtschaft und deren eigenen Ansatz zu Unternehmensführung, dessen Antriebskraft die gesellschaftliche Rentabilität wirtschaftlichen Handelns ist, in den verschiedenen Sektoren der Politik
Ermöglichung einer Stiftung im europäischen Rahmen sollten ebenso fortentwickelt werden wie die Erweiterung der europäischen Privatgesellschaft auf alle Unternehmen. Auch die nichtgewinnorientierten Unternehmen sollten europaweit gültige Handlungsformen erhalten. Das Europäische Parlament fordert sodann die Errichtung einzelstaatlicher statistischer Unternehmensregister sowie eine systematische Erfassung der sozialwirtschaftlichen Betätigungsfelder durch eigene Statistiken und spricht sich dafür aus, die Sozialwirtschaft als einen eigenen Sektor im Rahmen des europäischen sozialen Dialogs zu etablieren.

Weiter hebt das Europäische Parlament die wachstums- und beschäftigungsfördernde Wirkung der Sozialwirtschaft im Rahmen der

Lissabon-Strategie hervor. Die Sozialwirtschaft stärke den Unternehmergeist, schaffe qualitativ hochwertige Arbeitsplätze, befördere die soziale Wiedereingliederung und leiste damit einen originären Beitrag zur Überwindung der drei Unausgewogenheiten des Arbeitsmarktes: Arbeitslosigkeit, Instabilität der Arbeitsplätze und Ausgrenzung der Arbeitslosen aus der Gesellschaft. Die staatliche Unterstützung der Sozialwirtschaft sei als soziale Investition in die Entwicklung von Solidarnetzen $\mathrm{zu}$ verstehen und in diesem Zweck begründet. Die Sozialwirtschaft bewirke eine bessere Integration der Frauen in den Arbeitsmarkt, stabilisiere die von zyklischen Schwankungen geprägte Wirtschaft und richte wirtschaftliche Tätigkeit auf lokale Bedürfnisse aus.

\section{Fünf Forderungen}

Das Europäische Parlament regt an, Synergien bei der Entwicklung von Dienstleistungen zu suchen, im Rahmen des EG-Beihilfenrechts »der sozialwirtschaftlichen Realität Rechnung zu tragen «, fordert eine gerechte Förderung der kleinen und mittleren Unternehmen im Hinblick auf eine leichtere Zugänglichkeit zu Finanzhilfen auf mitgliedstaatlicher oder EU-Ebene und plädiert für eine gezielte Förderung der sozialwirtschaftlichen Tätigkeit sowohl bei der Gründung als auch dem Betrieb einzelner Einrichtungen durch Angebote von Information, Beratung und Weiterbildung. Schließlich ist anerkannt, dass Unternehmen der Sozialwirtschaft »mit mehr Schwierigkeiten zu kämpfen haben als Großunternehmen, beispielsweise bei der Bewältigung von Verwaltungsaufwand, ... Beschaffung von Finanzmitteln und beim Zugang zu neuen Technologien und Informationen «. Hieraus leitet das Europäische Parlament fünf Forderung ab:

Erstens: Die Kommission möge die Sozialwirtschaft im Rahmen ihrer Wirtschaftspolitik umfassend anerkennen und jene als wesentlichen Teil der europäischen wirtschaftlichen Entwicklung begreifen.

Zweitens: Die Mitgliedstaaten mögen sozialwirtschaftliche Organisationen anhalten, ihre Abhängigkeit von Beihilfen zu vermindern und ihre Nachhaltigkeit zu stärken.

Drittens: Die Kommission wird aufgefordert, die Vereinigungen der Sozialwirtschaft im Rahmen der Konsultations- und Dialogforen einzubeziehen.

Viertens: Gezielte Forschungen sollen auf das Gebiet der Sozialwirtschaft erstreckt werden.

Fünftens: Die Sozialwirtschaft soll das Thema namentlich in Ober- und 
Hochschulen behandelt, die Professionalisierung der Beschäftigten erhöht und Initiativen zur Schaffung eines einheitlichen Rechtsrahmens für sozialwirtschaftliche Tätigkeiten entfaltet werden.

\section{Würdigung}

Der »Bericht über die Sozialwirtschaft « vom 26. Januar 2009 des Ausschusses für Beschäftigung und soziale Angelegenheiten des Europäischen Parlaments ist für die europäische Sozialpolitik von weitreichender Bedeutung. In ihm findet die Sozialwirtschaft als eine innovative, dem Gemeinwohl verpflichtete Institution eine umfassende Anerkennung; ferner werden vielfältige Perspektiven für die Fortentwicklung des europäischen Rechts im Hinblick auf die Sozialwirtschaft formuliert; schließlich ergeben sich aus der Gesamtheit der gefundenen Einsichten und Handlungsempfehlungen erste markante Leitperspektiven zur Schaffung eines wünschenswerten und nach Inkrafttreten des Vertrages von Lissabon auch rechtlich gebotenen europäischen Rechtsrahmens für die Entfaltung sozialwirtschaftlicher Tätigkeiten.

\section{Umfassende Anerkennung sozial- wirtschaftlicher Tätigkeit}

Das Dokument erlangt seine prinzipielle Bedeutung zunächst aus der darin niedergelegten Einsicht in die

\section{Europäisches Parlament lobt Sozialwirtschaft}

Das Europäische Sozialmodell hat sich hauptsächlich durch ein hohes Niveau von Dienstleistungen, Produkten und Arbeitsplätzen sowie durch die von seinen Befürwortern unter Beweis gestellte Antizipations- und Innovationsfähigkeit herausgebildet, so das Europäische Parlament in einem Bericht vom Jahresanfang (A6-0015/2009 Sozialwirtschaft, REF 20090218IPR49792). Die Sozialwirtschaft stelle zehn Prozent aller europäischen Unternehmen und sechs Prozent der gesamten Beschäftigung. Sie habe ein hohes Potenzial zur Schaffung und Bewahrung stabiler Arbeitsplätze. »Die Sozialwirtschaft trägt zur Verwirklichung der vier Hauptziele der EU-Beschäftigungspolitik bei: Verbesserung der Beschäftigungsfähigkeit, Förderung des Unternehmergeists, Verbesserung der Anpassungsfähigkeit und Stärkung der Politik der Chancengleichheit, so die italienische Berichterstatterin Patrizia Toia. Die Sozialwirtschaft habe sich besonders durch Unternehmensformen wie Genossenschaften, Gegenseitigkeitsgesellschaften, Vereinigungen, Sozialunternehmen und Sozialorganisationen, Stiftungen und andere in jedem Mitgliedstaat existierende Formen entwickelt. Obwohl die Unternehmen der Sozialwirtschaft häufig als den Kapitalunternehmen gleichgestellt betrachtet werden, werden sie durch das Fehlen von Rechtsinstrumenten behindert. Die Sozialwirtschaft nehme bei der Verwirklichung der Lissabon-Ziele eine »Schlüsselrolle« ein, argumentiert das Europäische Parlament, da sie sich mit zahlreichen Ungleichgewichten auf dem Arbeitsmarkt befasst und so einen Beitrag zur örtlichen Entwicklung und zum sozialen Zusammenhalt leistet. Die Kommission wird aufgefordert, über die Wiederaufnahme einer der Sozialwirtschaft gewidmeten Haushaltslinie nachzudenken und Bedingungen anzustreben, die es ermöglichen, Investitionen in die Sozialwirtschaft zu erleichtern, insbesondere durch Investitionsfonds, durch die Gewährung von Darlehen und in Form von Subventionen. Die »Entschließung des Europäischen Parlaments vom 19. Februar 2009 zu der Sozialwirtschaft $(2008 / 2250$ (INI) « mit den Ergebnissen der Studie ist in deutscher Sprache auf der Website des Europäischen Parlaments veröffentlicht (Internet http://www.europarl.europa.eu/sides/getDoc.do? pubRef=-//EP//TEXT+TA+P6-TA-2009-0062+0+DOC+XML+V0// DE\&language $=\mathrm{DE})$.

Europäisches Parlament, Rue Wiertz - Wierzstraat, 1047 Brüssel, Telefon 0032 228-0, Fax 0032 22843530, E-Mail directinfo@europarl.eu.int, Internet http://www.europarl.eu.int

organisatorische und funktionelle Eigenständigkeit der Sozialwirtschaft als sowohl rechtlich wie wirtschaftlich eigenständige Teile der europäischen Wirtschaft. Daraus folgt die Einsicht, dass nicht alle Unternehmen gewinnorientiert ausgerichtet sind und demgemäß das EG-Wirtschaftsrecht mit seiner Betonung des Wettbewerbs, der freien Preisbildung und der prinzipiellen Zurückweisung jeder staatlicher Unterstützung nicht als die entscheidende Maß- und Richtgröße für sämtliche Unternehmen im Binnenmarkt sind und sein dürfen.

In der Anerkennung der Sozialwirtschaft als ein in der Wirtschaft eigenständiger Zweig liegt aber nicht nur die Billigung von deren Eigenständigkeit im Vergleich zu anderen Unternehmen. Zugleich liegt darin das Bekenntnis, dass die zentralen Bauelemente der Sozialwirtschaft namentlich deren soziale und Gemeinwohlorientierung, ihre Einbindung in die demokratische Kontrolle sowie ihre Unabhängigkeit vom Staat - einen Eigenwert darstellen, der die Sozialwirtschaft im Hinblick auf Verbraucher- und Mitarbeiterbelange auch zu einem Modell für die erwerbswirtschaftlich und gewinnorientierten Unternehmungen macht.

Damit ist der Sozialwirtschaft der Durchbruch als eigenständiger und die soziale Ausrichtung der europäischen Wirtschaft unterstreichender Zweig der wirtschaftlichen Ordnung gelungen. Künftige Debatten um den gemeinschafts- oder EU-rechtlich zu vollendenden Rechtsrahmen für die wirtschaftliche Betätigung von Unternehmen im Binnenmarkt werden und dürfen hinter diesen Erkenntnisstand nicht mehr zurückfallen.

\section{Perspektiven für die \\ Fortentwicklung des Europäischen Rechts im Hinblick auf die Sozialwirtschaft}

Die substanziellen Aussagen trifft der Bericht im Hinblick auf die Stellung der Sozialwirtschaft im EG-Wirtschaftsrecht. Von besonderem Interesse ist hierbei die Anerkennung ihrer Sonderstellung innerhalb des Kartellund Beihilferechts. Dies lässt sich namentlich daraus erklären, dass die Entgelte für sozialwirtschaftliche Leistungen statt nach dem für gewinn- 
orientierte Erwerbswirtschaft maßgeblichen Prinzips der freien Preisbildung öffentlich festgesetzt und oder auf der Grundlage der durchschnittlich anfallenden Aufwendungen ausgeglichen werden.

Im Hinblick darauf erfüllt das Kartellrecht im Rahmen der Erbringung sozialwirtschaftlicher Leistungen keine sinnvolle Funktion, denn es soll die freie Preisbildung ermöglichen. Des weiteren ist allgemein durch Beihilferecht zu gewährleisten, dass staatliche Zuwendungen selektiv an einzelne Unternehmen gehen und damit den Wettbewerb insgesamt verfälschen. Denn falls - wie in der Sozialwirtschaft - der Wettbewerb nicht wesentlich über den Preis, sondern über die vom Nachfrager perzipierte Güte der Leistungen stattfindet, sind staatliche Transfers an sozialwirtschaftliche Akteure nicht prinzipiell anstößig. Im Gegenteil, sie erlauben das Wirken mehrerer miteinander konkurrierender freier Träger und sichern bei der Erbringung sozialer Dienstleistungen damit die Staatsferne und Trägervielfalt und gewährleisten damit letztlich das Wunsch- und Wahlrecht für die Berechtigten bei der Inanspruchnahme sozialer Leistungen.

Die Sonderstellung des gemeinnützigen Sektors erklärt und begründet sich also aus den Eigenheiten seiner Finanzierung. Seine Leistungen richteten sich nach den Kosten für deren Erbringung und nicht nach den Gewinnerwartungen von Investitionen. Das vorherrschende Motiv der Kalkulation von Entgelten ist die Kostendeckung und nicht die Gewinnerzielung. Alles in allem bestätigt das
Europäische Parlament damit die gemeinwirtschaftliche Wirtschaft in ihrer gegenüber erwerbswirtschaftlichem Handeln kennzeichnenden Eigenheit wie Eigenständigkeit.

Wegen der ausgeprägten Dienstleistungsorientierung sozialwirtschaftlicher Tätigkeiten und ihres hohen lokalen Bezuges gehen von ihr ferner nicht nur erhebliche Beschäftigungswirkungen, sondern auch beträchtliche soziale und regionale Verteilungswirkungen aus. Deshalb sichern diese Unternehmen Teilhabechancen für die auf dem Arbeitsmarkt vom Risiko der Verdrängung bedrohten Gruppen der Erwerbsbevölkerung und fördern die gleichmäßige wirtschaftsstrukturelle Entwicklung aller Räume im Binnenmarkt.

Die Eigenständigkeit und hieraus folgende Vielfalt in den verschiedenen Mitgliedstaaten der Rechtsformen sozialwirtschaftlicher Betätigung hat in der EG eine beträchtliche rechtliche Vielfalt zur Folge. In einem auch die sozialwirtschaftlichen Leistungen umfassenden Binnenmarkt sind diese Verschiedenheiten grundsätzlich rechtlich anzuerkennen. Ein zu hohes Maß an Unterschiedlichkeit in der rechtlichen Ausgestaltung einzelner Handlungsformen führt jedoch auf dem Binnenmarkt zu Rechtsunsicherheit, weil Kenntnisse über die sozialwirtschaftlichen Organisationsregeln anderer Mitgliedstaaten in der Europäischen Union und den einzelnen Mitgliedstaaten nicht als bekannt vorausgesetzt werden können.

Es erscheint vor diesem Hintergrund sachgerecht, die in dem Bericht angedeuteten Bemühungen zu verstärken, die Rechtsformen sozialwirtschaftlicher Betätigung in der Europäischen Union einander anzunähern und - vor allem - für die europaweit tätige sozialwirtschaftliche Einrichtungen in Anlehnung an entsprechende Vorbilder für gewinnorientierte Unternehmen eine europäische Rechtsform für gemeinwirtschaftliches Handeln zu schaffen.

\section{Sozialwirtschaft und Universaldienste}

Die Initiative des Europäischen Parlaments über die Sozialwirtschaft stellt überraschenderweise $\mathrm{zu}$ wenig Bezüge her zu den eingangs skizzierten institutionellen Regeln, welche im geltenden oder künftigen Primärrecht für die Universaldienstleistungen getroffen sind. Weil das künftige Primärrecht dem Europäischem Parlament und Rat aufgeben, für das Wirken der Universaldienste Grundprinzipien und Ausrichtungen zu umschreiben, und die Sozialwirtschaft in diesem Kontext ihren Auftrag, ja ihre Erfüllung findet, erschiene es sachgerecht, wenn in den künftigen Beratungen über einen Rechtsrahmen für die Sozialwirtschaft auch eine rechtliche Ausgestaltung der Universaldienste aufgenommen würde und beide in ihrer wechselseitigen Bedingtheit füreinander erkannt werden würden.

Diese Überlegung macht deutlich, dass der Bericht über die Sozialwirtschaft zugleich einen wesentlichen Beitrag für die Fortentwicklung des Rechts der Universaldienstleistungen im Binnenmarkt darstellt. 